Lutfu S. Onderoglu • Mehmet Coskun Salman •

Ozgur Ozyuncu • Gurkan Bozdag

\title{
Successful management of a cornual pregnancy with a single high-dose laparoscopic methotrexate injection
}

Published online: 16 November 2005

(C) Springer-Verlag Berlin/Heidelberg 2005

\begin{abstract}
Cornual (interstitial) pregnancy, a very rare form of ectopic pregnancy, accounts for $2-4 \%$ of all tubal pregnancies in which conception is located in the proximal interstitial portion of the fallopian tube. Cornual resection by laparotomy is generally the preferred method of treatment, although more conservative approaches have also been described. We report a case of cornual pregnancy that was successfully treated with a single dose of $100 \mathrm{mg}$ methotrexate injected into the mass of the ectopic cornual pregnancy. The serum human chorionic gonadotropin (hCG) level decreased to undetectable levels on the 60th post-operative day.
\end{abstract}

Keywords Cornual pregnancy · Conservative management $\cdot$ Laparoscopy $\cdot$ Methotrexate injection

\section{Introduction}

Cornual (interstitial) pregnancy, a very rare form of ectopic pregnancy, accounts for $2-4 \%$ of all tubal pregnancies in which conception is located in the proximal interstitial portion of the fallopian tube [1]. Relative to other tubal pregnancies, cornual pregnancy tends to be diagnosed later in gestation, and it may often be associated with uterine rupture and massive intra-abdominal hemorrhage since the pregnancy occurs at a highly vascularized area. Consequently, the rate of maternal mortality is $2-2.5 \%$, which represents $20 \%$ of all deaths due to ectopic pregnancy [1,2]. Cornual resection by laparotomy is generally the preferred method of treatment, although more conservative approaches have also been described $[3,4]$.

L. S. Onderoglu · M. C. Salman $(\bowtie) \cdot$ O. Ozyuncu $\cdot$ G. Bozdag Department of Obstetrics and Gynecology, Faculty of Medicine, Hacettepe University, Sihhiye, Ankara, 06100, Turkey

e-mail: csalman@hacettepe.edu.tr

Tel.: +90-312-3051801

Fax: $+90-312-3052315$
We report here a case of cornual pregnancy successfully treated with a local methotrexate injection given via the laparoscopic approach.

\section{Case report}

A 40-year-old woman presented with lower abdominal pain and vaginal bleeding after an amenorrheic period of 6 weeks. Her past medical history was unremarkable except for two cesarean sections. An ultrasonographic examination revealed an eccentrically located gestational sac with a separate empty uterine cavity (Fig. 1a). The serum human chorionic gonadotropin (hCG) level was found to be $52,000 \mathrm{mIU} / \mathrm{ml}$. A decision for a laparoscopic evaluation was made, and laparoscropy revealed an unruptured cornual pregnancy on the right side (Fig. 1b). A single dose of $100 \mathrm{mg}$ methotrexate was injected into the mass of ectopic cornual pregnancy without any complications (Fig. 1c), and the patient was discharged from hospital on the same day with an uneventful post-operative course. On postoperative follow-up, the serum hCG level decreased to undetectable levels by the 60th post-operative day (Fig. 2).

\section{Discussion}

A cornual pregnancy has the potential to create life-threatening conditions since the implantation site is very close to major vessels and invasion through the uterine wall may cause a sudden and massive intra-abdominal bleeding following uterine rupture. Therefore, a timely diagnosis may often be life-saving. However, although the symptoms of cornual pregnancy are similar to those of other ectopic pregnancies, the patients are generally diagnosed later in gestation because of the better protection of the gestational sac in the interstitial tubal portion compared with other tubal portions [1].

Although ultrasonography is a useful diagnostic tool in the presence of a positive serum hCG test, laparoscopy is 

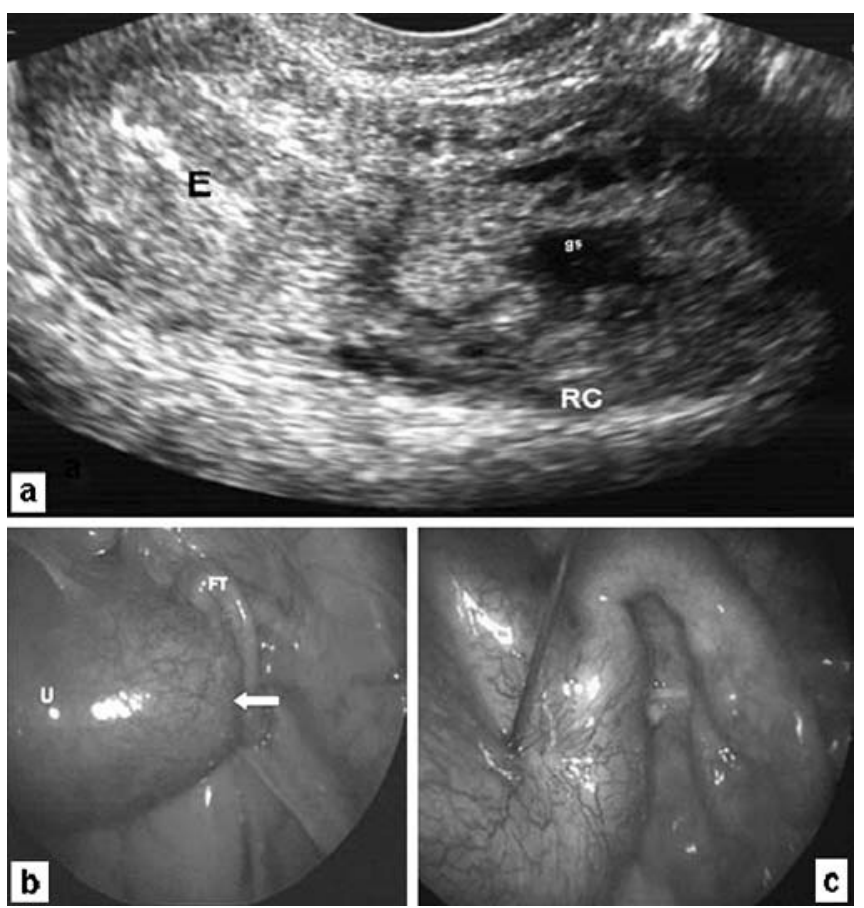

Fig. 1 a Preoperative ultrasound examination of the patient revealed an eccentrically located gestational sac with an empty uterine cavity. $E$ Endometrium, $R C$ right cornu, $g s$ gestational sac. b Unruptured right-sided cornual pregnancy (white arrow) was observed on laparascopy. $U$ uterus, $F T$ fallopian tube. c Injection of methotrexate with a $20 \mathrm{G}$ spinal needle

often required to confirm the diagnosis of cornual pregnancy $[1,3]$. Nevertheless, an immediate laparotomy should be performed in patients having a clinic picture consistent with heavy intra-abdominal bleeding.
The treatment choice depends on both the patient's hemodynamic status and future fertility desire. Traditional treatment approaches, especially in patients without an early diagnosis, are cornual resection with primary repair of the defect through laparotomy and hysterectomy in cases of hemodynamic instability or in patients with technical difficulties with respect to cornual repair [1]. Cornual resection may also be performed through laparoscopy [3]. However, in patients treated with cornual resection, the major risk is the possibility of uterine rupture in subsequent intrauterine pregnancies, and a careful antenatal follow-up should be offered to such patients [4].

When an unruptured cornual pregnancy is diagnosed, methotrexate may also be used either by systemic or local injection, or a combination of both. Although the overall success rate of methotrexate treatment with different regimens is $83 \%$, the success rate of local injection under laparoscopic or ultrasonographic guidance is slightly higher than that of systemic injection (91 and 79\%, respectively) [4]. Also, local injection results in fewer side effects because of a reduced systemic distribution of methotrexate [5]. Therefore, local injection under laparoscopy was the method preferred for our patient. The usual amount of methotrexate given as a single dose has been up to $1 \mathrm{mg} / \mathrm{kg}$ or $50 \mathrm{mg} / \mathrm{m}^{2}$. However, our patient was treated with a single dose of $100 \mathrm{mg}$ since a single local administration of methotrexate at a relatively high dose of $100 \mathrm{mg}$ has been shown to be both safe and effective in the medical management of relatively advanced and unselected ectopic pregnancies [6].

In conclusion, a conservative therapeutic approach such as the one described here may be considered under close follow-up in hemodynamically stable patients with future fertility desires, with surgical management reserved only for cases of failure. Laparoscopy, in addition to confirming the diagnosis, also provides the means of treatment at the same time without major morbidity.
Fig. 2 The hCG levels before and after methotrexate injection

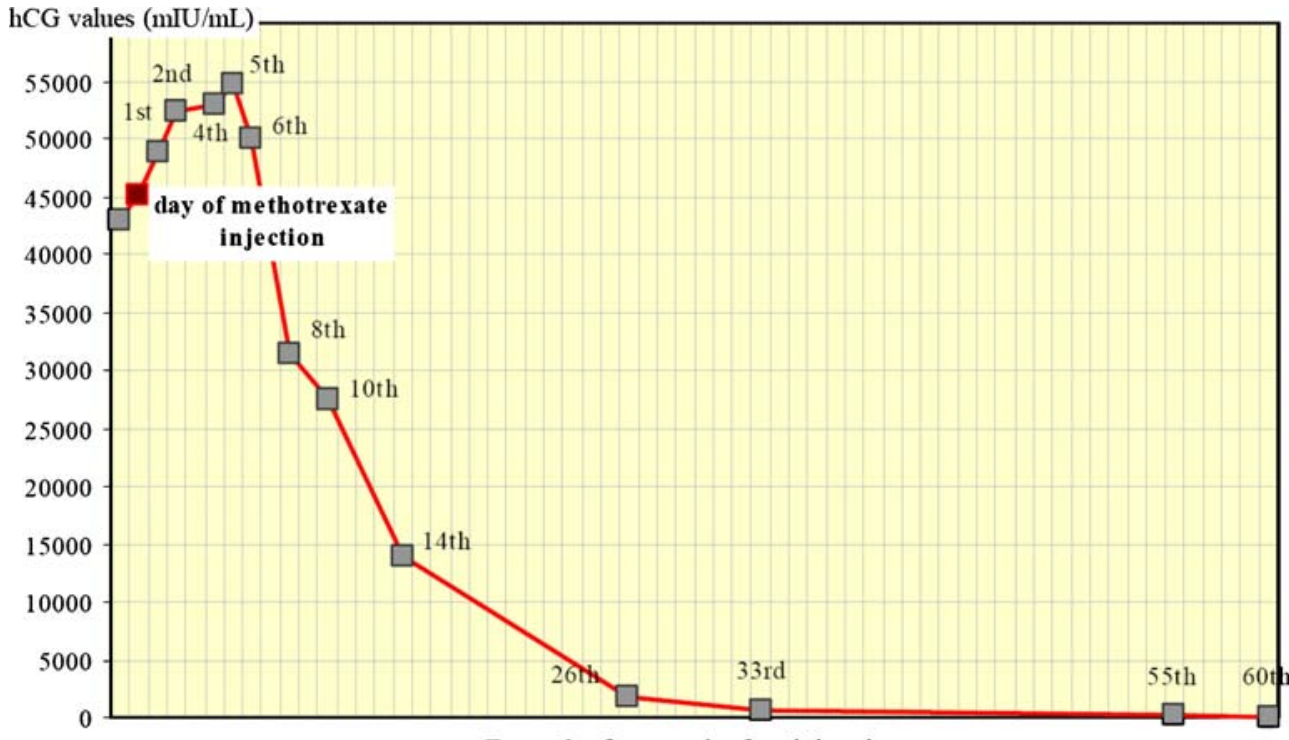

Days before and after injection 


\section{References}

1. Rock J (1997) Te Linde's operative gynecology. LippincottRaven, Philadelphia

2. Dorfman SF, Grimes DA, Cates W Jr, Binkin NJ, Kafrissen ME, O'Reilly KR (1984) Ectopic pregnancy mortality, United States, 1979 to 1980: clinical aspects. Obstet Gynecol 64:386390

3. Gezer A, Mutlu H (2004) Laparoscopic management of cornual pregnancy without sutures. Arch Gynecol Obstet 270:194-196
4. Lau S, Tulandi T (1999) Conservative medical and surgical management of interstitial ectopic pregnancy. Fertil Steril 72:207-215

5. Schiff E, Shalev E, Bustan M, Tsabari A, Mashiach S, Weiner E (1992) Pharmacokinetics of methotrexate after local tubal injection for conservative treatment of ectopic pregnancy. Fertil Steril 57:688-690

6. Tzafettas JM, Stephanatos A, Loufopoulos A, Anapliotis S, Mamopoulos M, Kalogeropoulos A (1999) Single high dose of local methotrexate for the management of relatively advanced ectopic pregnancies. Fertil Steril 71:1010-1013 